Cite this: Phys. Chem. Chem. Phys., 2013,

\title{
Chemically-synthesised, atomically-precise gold clusters deposited and activated on titania $\dagger$
}

15, 3917

\author{
David P. Anderson, ${ }^{a}$ Jason F. Alvino, ${ }^{\mathrm{b}}$ Alexander Gentleman, ${ }^{\mathrm{b}}$ Hassan Al Qahtani, ${ }^{\mathrm{c}}$ \\ Lars Thomsen, ${ }^{d}$ Matthew I. J. Polson, ${ }^{a}$ Gregory F. Metha, ${ }^{\star b}$ Vladimir B. Golovko*a \\ and Gunther G. Andersson*c
}

Received 11th November 2012, Accepted 16th January 2013

DOI: $10.1039 / \mathrm{c} 3 \mathrm{cp} 44005 \mathrm{~b}$

www.rsc.org/pccp

\begin{abstract}
Synchrotron XPS was used to investigate a series of chemically-synthesised, atomically-precise gold clusters $\mathrm{Au}_{n}\left(\mathrm{PPh}_{3}\right)_{y}(n=8,9,11$ and 101, with y depending on cluster size) immobilized on titania nanoparticles. The gold clusters were washed with toluene at $100{ }^{\circ} \mathrm{C}$ or calcined at $200{ }^{\circ} \mathrm{C}$ to remove the organic ligand. From the position of the $\mathrm{Au} 4 \mathrm{f}_{7 / 2}$ peak it is concluded that cluster size is not altered through the deposition. From the analysis of the phosphorous spectra, it can be concluded that the applied heat treatment removes the organic ligands. Washing and calcination leads to partial oxidation and partial agglomeration of the clusters. Oxidation of the clusters is most likely due to the interaction of the cluster core with the oxygen of the titania surface after removal of ligands. The position of the Au $4 f_{7 / 2}$ peak indicates that the size of the agglomerated clusters is still smaller than that of $\mathrm{Au}_{101}$.
\end{abstract}

\section{Introduction}

Fabrication of support-immobilized nanoparticles as model systems $^{1,2}$ in heterogeneous catalysis follows two distinct routes. The first route relies on the classical surface science UHV techniques. In this case, metal nanoparticles are created on flat supports either via gas phase delivery and support-assisted self-assembly ${ }^{3}$ of metal atoms by evaporation of the metal ${ }^{4-6}$ or by the deposition of size-selected clusters formed in the gas phase after the evaporation of the metal. ${ }^{7-9}$ The second route involves chemical synthesis of nanoparticles or their precursors followed by their deposition onto the support. This route offers the huge benefit of easy scale-up and testing of the fabricated materials under industrially relevant conditions. Generally, there are three alternative methods for the chemical catalyst fabrication route, with varying degree of control over the precision with which the nanoparticles are created. The simple chemical methods aim to

\footnotetext{
${ }^{a}$ The MacDiarmid Institute for Advanced Materials and Nanotechnology, Department of Chemistry, University of Canterbury, Christchurch 8140, New Zealand. E-mail: vladimir.golovko@canterbury.ac.nz

${ }^{b}$ Department of Chemistry, University of Adelaide, 5005, Australia. E-mail: greg.metha@adelaide.edu.au

${ }^{c}$ Flinders Centre for NanoScale Science and Technology, Flinders University, Adelaide SA 5001, Australia. E-mail: gunther.andersson@flinders.edu.au

${ }^{d}$ Australian Synchrotron, 800 Blackburn Road, Clayton Vic-3168, Australia

† Electronic supplementary information (ESI) available: CCDC 907703 and 907704. For ESI and crystallographic data in CIF or other electronic format see DOI: $10.1039 / \mathrm{c} 3 \mathrm{cp} 44005 \mathrm{~b}$
}

control the growth of nanoparticles on various supports via reduction/decomposition of simple precursors, such as simple metal salts or simple organometallic compounds. ${ }^{10}$ Improved control can be achieved via the immobilization of pre-made metal ion containing templates, such as metal ion containing dendrimers, ${ }^{11}$ onto supports followed by careful reduction/ decomposition to form well defined nanoparticles. Alternatively, the metal core (containing metal-metal bonds) of the nanoparticle can be formed during the synthesis stage (i.e. in the liquid phase $c f$. on the support surface) with an excellent degree of control over the size and chemical composition. Liquid phase synthesis of gold colloids with narrow metal core size distributions, stabilized by capping groups dates back to the 19th century studies by Faraday. ${ }^{12}$

Immobilization of colloids and clusters onto supports is an important step in the fabrication of heterogeneous catalysts. The post-fabrication activation of such catalysts by calcination ${ }^{13-17}$ or washing ${ }^{18}$ could afford nanoparticles, from which some or all ligands have been removed and where the size is defined by the specific precursor (colloid or cluster). However, in some cases catalytic activity is attributed to intact clusters on the support, i.e. clusters from which ligands have not been removed. ${ }^{19}$ Detailed studies of the effects of the activation processes on the morphology and electronic properties of the supportimmobilized nanoparticles are required to understand the nature of such catalysts. Importantly, support-immobilization of atomically-precise chemically-synthesised clusters offers an opportunity to control the active site of the heterogeneous 
catalyst with an ultimate precision allowing correlation of any observed catalytic reactivity with the structural and electronic properties of the key ingredients - exactly defined metal moieties. Thus, detailed understanding of the deposition of atomically precise gold clusters and the effect of activation processes is important for the development of better strategies for fabrication of heterogeneous catalysts.

Titania is a particularly important support material in the field of gold nanoparticle-based heterogeneous catalysts. ${ }^{3}$ Surprisingly, fabrication and activation of catalysts using chemically-synthesised, atomically precise gold clusters supported on titania is practically unexplored. ${ }^{20}$ Thus, for the purpose of this study we have focused our efforts on gold clusters supported on $\mathrm{TiO}_{2}$ P-25 Aeroxide - a commercially available material which is often used as a standard in the field of $\mathrm{TiO}_{2}$-focused research.

Gold particles with a size reduced below 5 nanometres are known to be catalytically active. ${ }^{21,22}$ Moreover, it was demonstrated that the onset of catalytic activity of gold coincides with the loss of metallic properties of Au nanoparticles at around 1.5-2 nm. This size-threshold is established for both naked clusters prepared under UHV conditions ${ }^{23}$ and chemically synthesised, ${ }^{14}$ well-defined metal nanoparticles immobilized onto inert supports.

More recently, there have been reports that the reactivity properties can become even more dramatic when the particle size is less than $1 \mathrm{~nm}$, where each particle can be considered as a nanocluster (or cluster). There is evidence of excellent catalytic performance of specific, size-selected clusters produced in the gas phase. For example, the $\mathrm{CO}$ oxidation by $\mathrm{Au}_{8}$ clusters deposited on $\mathrm{MgO}$ surfaces has been experimentally shown to occur at $150 \mathrm{~K},{ }^{9}$ whereas smaller clusters are inert. Hutchings and co-workers have demonstrated that a tiny amount of gold nanoclusters consisting of $c a .10$ atoms is responsible for high CO-oxidation activity of a catalyst containing a majority of gold in the form of larger particles immobilized on iron-oxide. ${ }^{24}$ Similarly, Flytzani-Stephanopoulos and co-workers have shown that sub-1 nm gold particles on ceria are highly active in the methanol-steam-reforming and water-gas-shift reactions, compared to effectively inactive particles larger than $3 \mathrm{~nm}$ in size. ${ }^{25,26}$ Finally, chemically made, atomically-precise gold clusters stabilized by thiols (i.e. strongly bonding ligands) have very recently been shown to be active in reactions of oxidation and hydrogenation. ${ }^{27-29}$

Whilst there is general agreement that the size and the electronic structure of the active components of the nanoparticles plays a critical role in the catalytic process, systematic studies revealing the electronic structure of chemically-synthesized gold clusters used in catalysis are still very rare. For this reason, electronic spectroscopy, such as XPS, is an important tool in identifying the electronic effects at play. ${ }^{30}$ In particular, many studies have focussed on the $4 f_{7 / 2}$ orbital of gold as it provides a convenient and sensitive measure of the electronic state of gold. In the following five paragraphs we will give an overview of how the binding energy of the core electrons determined by XPS informs about the size and chemical state of the deposited clusters. This overview is essential for the interpretation of the XPS spectra. However, the reader could also proceed to the end of this section where a summary is provided.
Firstly, the oxidation state plays a major role in the peak position. The literal gold standard is bulk gold (i.e. $\mathrm{Au}^{0}$ ), in which the $4 \mathrm{f}_{7 / 2}$ peak is well known to appear at $84.0 \mathrm{eV}$ with a $3.67 \mathrm{eV}$ separation to the $4 \mathrm{f}_{5 / 2}$ peak. Upon complete oxidation to $\mathrm{Au}_{2} \mathrm{O}_{3}$, the $4 \mathrm{f}_{7 / 2}$ peak shifts to 85.8-86.2 eV, depending upon the substrate ${ }^{31,32}$ the thickness of gold oxide layer is estimated to be between $0.75 \mathrm{~nm}^{31}$ and $3.3 \mathrm{~nm},{ }^{32}$ providing the $4 \mathrm{f}_{7 / 2}$ peak position values for a macroscopic layer of gold oxide surface ( $c f$. nanoparticles of $\mathrm{Au}_{2} \mathrm{O}_{3}$ ). Secondly, initial and final states effects also determine the peak position as well as the fullwidth-half-maximum of the peaks, both depending on the element forming the cluster, its size and its interaction with the substrate. ${ }^{33}$ Changes in the valence electron structure resulting from reduced size of the cluster ( $c f$. the bulk metal) and changes due to the interaction with the substrate contribute to the initial state effects. ${ }^{33}$ After excitation, the metal nanocluster can stay in a charged state for a certain period of time, which often depends on the size of the cluster and the type of the substrate. This is often referred to as a final state effect. $^{33}$ Therefore, the peak position and width can provide information that is of fundamental importance for understanding the electronic structure of metal nano-clusters, whether they are prepared via gas phase deposition or are chemically synthesised. However, interpreting changes of peak positions as an initial or final state effect based on experimental data is very difficult for gold as the Auger lines are rather weak. ${ }^{33,34}$ Thus, in the present work only changes in peak positions will be considered and not interpreted as initial or final state effects.

Over the last decade there have been a number of studies of sub-monolayer gold films evaporated onto various substrates and of chemically synthesised clusters in various matrices. A large variety of results was reported. The difficulty with finding a unifying thread between these studies is the uncertainty in particle size, particularly below $1 \mathrm{~nm}$, where exact size determination is difficult. We provide a thorough, though not exhaustive, description of previous work using XPS to determine key properties of ultra-small gold particles/clusters. It is ordered according to the specific way in which the materials have been fabricated. We will start with gold nanoparticles within the ultra-small (sub-2 nm) particle size-regime made without precise control of the particle sizes; followed by atomically precise naked gold clusters made under UHV conditions, size-selected and soft-landed onto specific support material; and finally discuss the atomically-precise, chemically-synthesised ligand protected gold clusters deposited onto a specific support from solution.

Büttner et al. evaporated gold onto pristine HOPG and defect-rich HOPG following bombardment with Ar ions. No peak shifts were found on the pristine surface, whereas gold on the defect-rich HOPG shows peak shifts of up to $+1.5 \mathrm{eV}$, multiple peaks and peak broadening. ${ }^{4}$ The authors attribute the peak shift and broadening to the preferential formation of gold particles at the defect sites which are found in a much higher number on the HOPG exposed to Ar bombardment. Kitsudo et al. evaporated gold onto graphite and various metal oxides. ${ }^{5}$ The gold peaks did not shift on the graphite and $\mathrm{NiO}(001)$ substrates. However, peak shifts of up to $+0.4 \mathrm{eV}$ were 
observed on $\mathrm{SrTiO}_{3}, \mathrm{Al}_{2} \mathrm{O}_{x} / \mathrm{NiAl}(110), \mathrm{TiO}_{2}$ and $\mathrm{NiO}(111)$ at submonolayer gold coverage. Importantly, on both $\mathrm{SrTiO}_{3}$ and graphite the full-width-half-maximum (FWHM) of the gold peak decreased slightly with increasing particle size. Lykhach et al. evaporated gold on $\mathrm{Nb}_{2} \mathrm{O}_{5} / \mathrm{Nb}, \mathrm{Nb}_{2} \mathrm{O}_{5} / \mathrm{W}$ and $\mathrm{NbO} / \mathrm{Nb}$ surfaces. ${ }^{35}$ They find shifts in the gold peak position of up to $+0.7 \mathrm{eV}$, with the largest shifts observed for the smallest $\mathrm{Au}$ coverage. Lim et al. evaporated gold on $\mathrm{SiO}_{2} / \mathrm{Si}$ and observed shifts of the gold peak of up to $+1 \mathrm{eV}$. Again, the largest shift corresponded to the smallest amount of gold evaporated. Exposure to atomic oxygen leads to the appearance of a second peak at about $86 \mathrm{eV}$, attributed to the formation of $\mathrm{Au}_{2} \mathrm{O}_{3}{ }^{6}$

Deposition of size-selected clusters produced under gasphase conditions have been known for over a decade. DiCenzo et al. measured Au $4 \mathrm{f}$ XPS data for gas phase, size-selected $\mathrm{Au}_{n}$ clusters deposited on amorphous carbon. For cluster sizes of $n=5,7,27$ and 33 they observed shifts of $+0.7,+0.6,+0.5$ and $+0.5 \mathrm{eV}$, respectively, with peak FWHM being slightly broader for the smaller clusters. ${ }^{36}$ Cox et al. reported Au $4 f_{7 / 2}$ XPS data for gas phase, size-selected positively charged clusters deposited on silica (natural oxide layer on $\mathrm{Si}(100)$-wafers). They observed a shift of $+0.8-1.0 \mathrm{eV}$ for $\mathrm{Au}_{n}(n=1-7) .{ }^{37}$ More recently, Lim et al. have published a series of papers where massselected $\mathrm{Au}$ cluster anions up to 20 atoms were soft-landed onto $\mathrm{SiO}_{2} / \mathrm{Si}$ surfaces and the samples subsequently exposed to atomic oxygen. ${ }^{6,38-40}$ Although peak widths of $1.5 \mathrm{eV}$ were observed, in addition to $\mathrm{Au}^{0}$ peaks, the clusters also exhibited features attributed to $\mathrm{Au}^{\mathrm{III}}$, which were shifted by $+1.5 \mathrm{eV}$. Interestingly, an even-odd effect was observed, whereby the odd-numbered clusters were inert towards oxidation and exhibited very small $\mathrm{Au}^{\mathrm{III}}$ peaks, particularly so for $\mathrm{Au}_{5}, \mathrm{Au}_{7}$ and $\mathrm{Au}_{13}$. These workers also deposited the same clusters on sputterdamaged HOPG. In this case, only $\mathrm{Au}_{8}$ showed any ability to oxidise after oxygen treatment. Small gold peak shifts of $+0.1 \mathrm{eV}$ to $+0.2 \mathrm{eV}$ were observed relative to bulk gold.

XPS studies have also been performed on chemically-synthesised, ligand protected gold species; from monoatomic $\mathrm{Au}$ compounds (e.g. $\left.\mathrm{Au}\left(\mathrm{PPh}_{3}\right)\left(\mathrm{NO}_{3}\right)\right)$, to atomically precise Au clusters (e.g. $\left.\mathrm{Au}_{8}\left(\mathrm{PPh}_{3}\right)_{8}\left(\mathrm{NO}_{3}\right)_{3}\right)$, to even larger, less precise, nanoparticles spanning the size range from $c a .1 .5 \mathrm{~nm}\left(\right.$ " $\left.\mathrm{Au}_{101}{ }^{, 41}\right)$ and above. Yuan et al. have deposited $\mathrm{Au}\left(\mathrm{PPh}_{3}\right)\left(\mathrm{NO}_{3}\right)$ on $\mathrm{Ti}(\mathrm{OH})_{4}$ and $\mathrm{TiO}_{2}$ showing a $\mathrm{Au} 4 \mathrm{f}_{7 / 2}$ of $85.5 \mathrm{eV}$ in the first case and $84.8 \mathrm{eV}$ in the latter case and attributed the difference in binding energy to different interactions of the cluster with the substrate. ${ }^{42}$ Chusuei et al. deposited $\left[\mathrm{Au}_{6}\left(\mathrm{PPh}_{3}\right)_{6}\right]\left[\mathrm{BF}_{4}\right]_{2}$ clusters on $\mathrm{TiO}_{2}$ and observed $\mathrm{Au} 4 \mathrm{f}_{7 / 2}$ peaks with binding energy of $83.9 \mathrm{eV} .{ }^{43}$ Removal of the triphenylphosphine ligand groups via electron-stimulated desorption resulted in a shift of $+0.4 \mathrm{eV}$. This was attributed to the removal of the ligands, which was corroborated by a reduction in size as observed by STM. Very early XPS studies by Battistoni et al. investigated $\mathrm{Au}_{1} \mathrm{Au}_{4}, \mathrm{Au}_{8}, \mathrm{Au}_{9}$ and $\mathrm{Au}_{11}$ clusters stabilised by triphenylphosphine ligands. ${ }^{4,45}$ Samples deposited from alcohol solutions onto steel sample plates showed $\mathrm{Au}$ binding energies that were 0.7 to $1.7 \mathrm{eV}$ higher than bulk gold, and which depended upon the number of ligands and the counter ions. By analysis of the different peak shapes they concluded that $\mathrm{Au}_{9}$ and $\mathrm{Au}_{11}$ clusters possess centred polyhedral geometries with a central metal atom involved only in metal-metal bonds. Van Attekum and co-workers similarly published an XPS analyses of chemically synthesised $\mathrm{Au}_{8}, \mathrm{Au}_{9}$ and $\mathrm{Au}_{11}$ stabilised by tri-arylphosphine ligands. ${ }^{46}$ Clusters were evaporated onto graphite substrates from solutions in dichloromethane. All clusters exhibited individual Au $4 f_{7 / 2}$ peaks with widths of $1.5 \mathrm{eV}$. Only kinetic energy values have been tabulated and calibrated relative to the $\mathrm{P}$ peak, however, these could be subject to a chemical shift so the peak positions may not be reliable. Boyen et al. used XPS to investigate a series of gold nanoparticles made using polymers as stabilizing agents with metal core sizes ranging from 2.9 down to $0.8 \mathrm{~nm}$ deposited on silicon. Following removal of stabilizers by treatment first with oxygen and subsequently with hydrogen plasma, the authors found peak shifts of up to $+0.5 \mathrm{eV}$ at their smallest cluster size of $0.8 \mathrm{~nm} .{ }^{47}$ The same authors found that phosphine stabilized $1.4 \mathrm{~nm}$ (similar size as $\mathrm{Au}_{101}$ (ref. 41)) nanoparticles after oxygen/ hydrogen plasma treatment show an $\mathrm{Au} 4 \mathrm{f}_{7 / 2}$ peak at about $84.2 \mathrm{eV}$. Ono et al. reported for polymer-stabilised gold particles peak positions of about $85 \mathrm{eV}$ for $1.5 \mathrm{~nm}$ gold and about $87 \mathrm{eV}$ for oxidised $1.5 \mathrm{~nm}$ gold clusters on both $\mathrm{TiO}_{2}$ and $\mathrm{SiO}_{2}$ substrates following treatment at $150 \mathrm{~K}$ with oxygen plasma only. ${ }^{48}$ Upon heating, they observed very fast decomposition of gold oxide species (within $6 \mathrm{~min}$ at $350 \mathrm{~K}$ ) for the nanoparticles supported on $\mathrm{TiO}_{2}$ which was in stark contrast to the case of $\mathrm{SiO}_{2}$-supported gold oxide species. More recently, Turner et al. found the $\mathrm{Au} 4 \mathrm{f}_{7 / 2}$ peak of $1.5 \mathrm{~nm}$ gold clusters deposited on $\mathrm{BN}$ at a binding energy of $85.1 \mathrm{eV} .^{14}$

In summary, the smaller clusters tend to exhibit the highest binding energy shifts, with the binding energy decreasing as cluster size increases before finally converging to the bulk value at large cluster sizes. ${ }^{5,36,47,49,50}$ The FWHM also shows in many cases an increase with decreasing cluster size., ${ }^{5,35}$ The shift, however, also strongly depends on the substrate. For "inert" substrates such as HOPG and pure Si, only small shifts, $<0.1 \mathrm{eV}$, are observed. $\mathrm{SiO}_{2}$ surfaces tend to shift the peaks by $\sim 0.2 \mathrm{eV}$ to higher energy. Highly interacting surfaces such as $\mathrm{TiO}_{2}, \mathrm{SrTiO}_{3}$, $\mathrm{Al}_{2} \mathrm{O}_{3}$ and $\mathrm{NiO}(111)$ induce shifts up to $1.5 \mathrm{eV}$.

The goal of this paper is to identify changes in electronic structure for a series of chemically-synthesised, atomicallyprecise gold clusters that occur upon their deposition onto titania followed by selected treatments - washing and calcination under mild conditions. Using Synchrotron XPS we have monitored removal of ligands, changes attributed to partial agglomeration and interaction of the cluster core with the substrate.

\section{Experimental}

The clusters were synthesized according to well-established, previously reported methods. Each cluster has been recrystallised at least twice to achieve high purity. Furthermore, their identities have been confirmed by NMR, MS and X-ray crystallography (CCDC 907704 for $\mathrm{Au}_{11}$ and 907703 for $\mathrm{Au}_{8}$, Fig. 1 and ESI $\dagger$ ). Specific details of the synthesis and purification via recrystallisation of the $\mathrm{Au}_{n}$ clusters $(n=8,9,11,101)$ is described in the ESI. $\dagger$ 

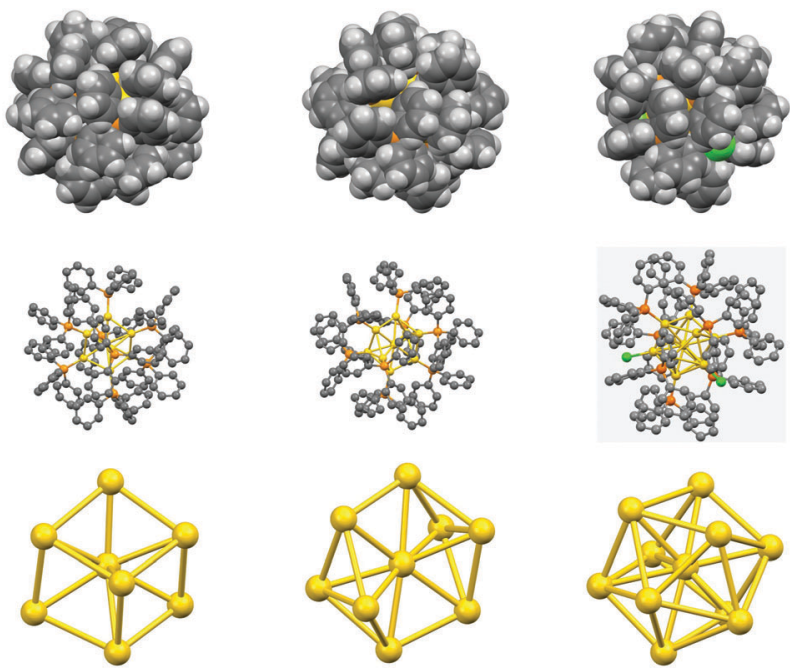

Fig. 1 Crystal structures of $\mathrm{Au}_{8}$ (left column), $\mathrm{Au}$ (centre column) and $\mathrm{Au}_{11}$ (right column) clusters showing the exact number of Au atoms per cluster core. Top row: space-filled models showing triphenylphospine ligands effectively protecting the metal cores buried underneath. Centre row: ball and stick model showing positions of all atoms except hydrogen, co-crystallisation solvent molecules and non-coordinated counter anions. Bottom row: geometries of the metal cores only. The atomic colour scheme is gold $(\mathrm{Au})$, orange $(\mathrm{P})$, green $(\mathrm{Cl})$, and black (C).

The following information regarding preparation of the supportimmobilised clusters and treatments is presented below because it is essential to the discussion.

\section{Deposition of clusters onto support}

A solution of the cluster $(20 \mathrm{mg})$ in methanol $(30 \mathrm{~mL})$ was added to the magnetically stirred suspension of acid washed Evonik P-25 (2 g) in methanol $(50 \mathrm{~mL})$ in a Schlenk tube. The suspension was left overnight and the solvent removed under vacuum taking care to avoid contamination of the Schlenk line with the resulting dry powder. The material obtained in this way was stored in a refrigerated Schlenk tube under $\mathrm{N}_{2}$ and in the absence of light.

Evonik P-25 particles were chosen as substrates as this material is a very popular $\mathrm{TiO}_{2}$ support used by numerous researchers worldwide, which very recently was U.S. National Institute of Standards and Technology as a nanoscale reference material. Further, particles have a large number of surface defects which is assumed to support the adsorption on the surface. ${ }^{51}$ It has to be noted that Evonik P-25 is a mixture of $70 \%$ anatase and $30 \%$ rutile particles. The interaction between the gold clusters and anatase and rutile could differ ${ }^{52}$ resulting in two different types of surfaces after deposition of the gold clusters. Prior to deposition of the gold clusters, the titania particles were treated with sulphuric acid. ${ }^{18}$

\section{Supported cluster treatment conditions}

Solvent treatment (washing) was undertaken following a similar protocol described by Lopez-Sanchez et al. ${ }^{18}$ Typically, the magnetically stirred (1000 rpm) suspension of the supported cluster on $\mathrm{TiO}_{2}(c a .500 \mathrm{mg})$ in toluene $(50 \mathrm{~mL})$ was heated to $100{ }^{\circ} \mathrm{C}$ for 2 hours. The solid was then recovered using centrifugation (10 $\mathrm{min}, 5000 \mathrm{rpm})$. The solid was then washed three times with toluene $(50 \mathrm{~mL})$ by forming a suspension using a Vortex agitator followed by centrifugation (10 $\mathrm{min}, 5000 \mathrm{rpm})$ to recover the solid, which was then dried under reduced pressure at about $10^{-2}$ mbar. The material obtained in this way was stored in a refrigerated Schlenk tube under $\mathrm{N}_{2}$ and in the absence of light.

Toluene was chosen as a solvent in the washing procedure as it is frequently used in catalytic processes. Thus, washing treatment with toluene at elevated temperatures also mimics the effect of the induction period during the first two hours if untreated catalyst is used.

\section{Heat treatment or calcination}

We follow the protocol described by M. Turner et al. ${ }^{14}$ The supported cluster on $\mathrm{TiO}_{2}(c a .500 \mathrm{mg}$ ) in a Schlenk tube was stirred (1000 rpm) and heated at $200{ }^{\circ} \mathrm{C}$ (oil bath) under vacuum for 2 hours. The sample was then removed from the oil bath and allowed to cool to room temperature under reduced pressure. The material obtained in this way was stored in a refrigerated Schlenk tube under $\mathrm{N}_{2}$ and in the absence of light.

\section{Sample preparation for XPS analysis}

A suspension of the support-immobilised cluster was made up in dichloromethane at a concentration of $c a .1 \mathrm{mg} \mathrm{mL}{ }^{-1}$. A $10 \mu \mathrm{L}$ drop of each sample was deposited onto a clean $6 \times 6 \mathrm{~mm}$ silicon ( $\mathrm{Si}$ ) wafer and dried in air. Each sample was then fixed by double-sided copper tape onto a gold-plate; up to 6 samples were affixed onto each plate for XPS analysis. On a representative number of samples, various spots were investigated. Between the investigated spots, no significant variation of the XPS signals was found. To confirm reproducibility of our results a completely new batch of the $\mathrm{Au}_{9}$ cluster was deposited on a new batch of acid washed $\mathrm{TiO}_{2}$ and the resulting material was heat treated as before. The XPS spectra for new "untreated" and "calcined" samples were in excellent agreement with earlier obtained data.

\section{Synchrotron beam line}

Photoelectron spectra were recorded at the Soft X-ray Beamline at the Australian Synchrotron (AS) using a SPECS Phoibos 150 hemispherical electron analyser with the photon energy set to $650 \mathrm{eV}$. The beam was adjusted to yield an irradiation spot size of $\sim 600 \times 600 \mu \mathrm{m}$, yielding an X-ray photon flux of approximately $10^{12}$ photons $\mathrm{mm}^{-2} \mathrm{~s}^{-1}$, conditions that have been shown not to induce thermal damage to samples of $\mathrm{NaAuCl}_{4} \cdot{ }^{53}$ High resolution XPS spectra of C, O, Si, P, Ti and $\mathrm{Au}$ were recorded at a pass energy of $10 \mathrm{eV}$, yielding an instrumental resolution of $295 \mathrm{meV} .{ }^{54}$ Scans were repeated several times to ensure that no photon-induced changes occurred in the samples. The stability of the energy of the $\mathrm{X}$-ray was monitored using a bulk gold reference.

All XPS spectra were fitted in the following manner. A Shirley background was applied to remove the electron-scattering background and maintain the intrinsic line shape from the raw data. ${ }^{55,56}$ 
Table 1 Position, FWHM and intensity of the Au-LBP and Au-HBP

\begin{tabular}{|c|c|c|c|c|c|c|c|c|c|}
\hline & \multicolumn{3}{|l|}{ Untreated } & \multicolumn{3}{|l|}{ Washed } & \multicolumn{3}{|l|}{ Calcined } \\
\hline & Energy $[\mathrm{eV}]$ & $\begin{array}{l}\text { Intensity rel. to } \\
\text { untreated }[\%]\end{array}$ & FWHM $[\mathrm{eV}]$ & Energy $[\mathrm{eV}]$ & $\begin{array}{l}\text { Intensity rel. to } \\
\text { untreated }[\%]\end{array}$ & FWHM [eV] & Energy $[\mathrm{eV}]$ & $\begin{array}{l}\text { Intensity rel. to } \\
\text { untreated [\%] }\end{array}$ & FWHM $[\mathrm{eV}]$ \\
\hline $\mathrm{Au}_{8} \mathrm{LBP}$ & $85.1 \pm 0.1$ & 100 & $1.6 \pm 0.2$ & $84.8 \pm 0.1$ & $37 \pm 10$ & $1.5 \pm 0.2$ & $84.0 \pm 0.1$ & $7 \pm 10$ & $1.2 \pm 0.2$ \\
\hline $\mathrm{Au}_{8} \mathrm{HBP}$ & - & - & - & $85.6 \pm 0.1$ & $16 \pm 20$ & $1.3 \pm 0.2$ & $85.4 \pm 0.1$ & $19 \pm 10$ & $1.6 \pm 0.1$ \\
\hline $\mathrm{Au}_{9} \mathrm{LBP}$ & $85.1 \pm 0.1$ & 100 & $1.6 \pm 0.2$ & $84.8 \pm 0.1$ & $47 \pm 10$ & $1.7 \pm 0.2$ & $84.2 \pm 0.1$ & $12 \pm 10$ & $1.3 \pm 0.2$ \\
\hline $\mathrm{Au}_{9} \mathrm{HBP}$ & - & - & - & $85.9 \pm 0.1$ & $17 \pm 10$ & $1.2 \pm 0.2$ & $85.5 \pm 0.1$ & $33 \pm 10$ & $1.5 \pm 0.1$ \\
\hline $\mathrm{Au}_{11} \mathrm{LBP}$ & $84.7 \pm 0.1$ & 100 & $1.6 \pm 0.2$ & $85.0 \pm 0.2$ & $13 \pm 20$ & $1.9 \pm 0.2$ & $84.2 \pm 0.1$ & $15 \pm 10$ & $1.1 \pm 0.2$ \\
\hline $\mathrm{Au}_{11} \mathrm{HBP}$ & - & - & - & $86.1 \pm 0.1$ & $18 \pm 10$ & $1.3 \pm 0.2$ & $85.5 \pm 0.1$ & $40 \pm 10$ & $1.5 \pm 0.1$ \\
\hline $\mathrm{Au}_{101} \mathrm{LBP}$ & $83.9 \pm 0.1$ & 100 & $1.0 \pm 0.2$ & $83.9 \pm 0.1$ & $5 \pm 5$ & $1.0 \pm 0.2$ & $83.9 \pm 0.1$ & $28 \pm 10$ & $0.9 \pm 0.2$ \\
\hline $\mathrm{Au}_{101} \mathrm{HBP}$ & - & - & - & - & - & & $84.8 \pm 0.1$ & $14 \pm 10$ & $2.3 \pm 0.2$ \\
\hline
\end{tabular}

A pseudo-Voigt function composed of the sum of Gaussian (30\%) and Lorentzian (70\%) functions was used to fit all peaks and all peak positions were allowed to vary using nonlinear least-squares minimization. ${ }^{57}$ For the $\mathrm{Au} 4 \mathrm{f}$ doublet, the splitting was fixed at $3.67 \mathrm{eV}$ while for the $\mathrm{P} 2 \mathrm{p}$ doublet a splitting of $0.84 \mathrm{eV}$ was used. ${ }^{58}$ All spectra where fitted with the least number of peaks allowing a variation of the FWHM, although the FWHM of a single contributing species was kept constant. For example, in the case of a gold spectrum fitted with two sets of $4 \mathrm{f}_{7 / 2}$ and $4 \mathrm{f}_{5 / 2}$ doublets, the FWHM within a single doublet was kept constant but was allowed to differ between the doublets.

The position, the intensity and the FWHM of the peaks fitted to the $\mathrm{Au} 4 \mathrm{f}_{7 / 2}$ spectra including the errors are given in Table 1. As an example, the fitting of the $\mathrm{Au}_{9}$ spectra are shown in the ESI. $†$

Cross sections were calculated according to Yeh and Lindau using the photoionization cross section and the asymmetry parameter. ${ }^{59}$ The angle between the sample normal and the analyser was $10^{\circ}$ which means that the angle between the incident horizontal linearly polarised synchrotron beam and the sample surface was $45^{\circ}$.

The preparation of reference samples and recording of XP spectra is described in the ESI. $\nmid$ The reference samples were prepared such that multilayers of untreated $\mathrm{Au}_{n}$ clusters were deposited on UHV compatible double-sided, adhesive copper conducting tape (3M brand). Due to this sample preparation, the reference XP spectra are recorded from $\mathrm{Au}_{n}$ clusters that are not in contact with a substrate, i.e. no cluster-substrate interaction occurs.

\section{Results and discussion}

The molecular clusters $(n=8,9,11)$ used in this study contain atomically precise, ideally monodisperse metal cores protected by phosphine ligands, while $\mathrm{Au}_{101}$ particles (also stabilised by phosphine ligands) have narrow particle size distribution centered around $1.5 \mathrm{~nm}$. The crystal structures of $\mathrm{Au}_{11}, \mathrm{Au}_{9}$ and $\mathrm{Au}_{8}$ clusters are shown in Fig. 1, confirming that the cluster metal core contain exactly $8,9^{60}$ and 11 atoms of gold respectively, with all the gold atoms involved in forming the metal-metal bonded cluster core. The two new X-ray structures reported here $\left(\mathrm{Au}_{11}\right.$ and $\left.\mathrm{Au}_{8}\right)$ show typical $\mathrm{Au}-\mathrm{Au}$ bonds of
2.6437(9) to 3.1274(10) $\AA$ in $\mathrm{Au}_{11}$, and 2.5827(6) to 2.8924(7) $\AA$ in $\mathrm{Au}_{8}$. The gold cluster at the centre of the $\mathrm{Au}_{8}$ structure approximates the cyclohexane structure with one gold atom added above and below the ring. This gives a shape with a short axis of 2.5827(7) $\AA$, and a distance across the ring that varies from 5.2533(5) to 5.4536(7) $\AA$. The gold cluster in the $\mathrm{Au}_{11}$ structure is quite spherical with ten ligated gold atoms (either by triphenylphosphine or chloride) surrounding a central gold atom. The shortest distance across the cluster 4.695(2) $\AA$ and the longest is 5.385(2) A. Further discussion of the XPS results will be carried out in the light of the atomic precision of the molecular cluster systems studied here.

Spectra have been taken at the gold $4 \mathrm{f}$, phosphorous $2 \mathrm{p}$, silicon 2p, titanium 2p, carbon 1s and oxygen 1s regions for $\mathrm{Au}_{n}$ clusters deposited on titania nanoparticles and from a gold reference sample. The majority of carbon on the samples is either carbon of the triphenylphosphine ligand of the gold cluster or adventitious hydrocarbons. In either case, we assigned the $\mathrm{C} 1 \mathrm{~s}$ peak to $285 \mathrm{eV}$ and used it for calibrating the peak positions of other elements. This is justified because adventitious hydrocarbons display a very constant $\mathrm{C} 1 \mathrm{~s}$ peak position and are present in all samples exposed to air (and not cleaned by sputtering etc. prior to XPS measurements). ${ }^{61}$ The titanium signal measured is exclusively due to the titania nanoparticles and found at $459.0 \pm 0.1 \mathrm{eV}$. The silicon signal is due to the silicon wafer used as substrate and found at $99.2 \pm$ $0.1 \mathrm{eV}(\mathrm{Si})$ and $103.1 \pm 0.1 \mathrm{eV}\left(\mathrm{SiO}_{2}\right)$. The oxygen signal is mostly due to titanium dioxide $(530.3 \pm 0.1 \mathrm{eV})$ and silicon dioxide $(532.4 \pm 0.1 \mathrm{eV})$. Gold signal due to the gold clusters is found between 83.7 and $86.1 \mathrm{eV}$. Phosphorous from the ligands is found at $131.8 \pm 0.1 \mathrm{eV}$ and $133.0 \pm 0.2 \mathrm{eV}$. The $\mathrm{Au}$ and $\mathrm{P}$ signals are used for quantitative analysis. Using the energy calibration described above, the $\mathrm{Au} 4 \mathrm{f}_{7 / 2}$ peak of bulk gold is found at a binding energy of $84 \mathrm{eV}$. This value is used to determine the shift between the $\mathrm{Au} 4 \mathrm{f}_{7 / 2}$ signal of the gold clusters and the bulk gold binding energy. Most references for gold are found at a binding energy of $84 \mathrm{eV}$ within a range of $\pm 0.2 \mathrm{eV} .^{62-65}$ The invariance of the titanium and silicon signals relative to the gold reference is less than $0.05 \mathrm{eV}$, indicating that there is no charging of the samples or instability in the $\mathrm{X}$-ray energy. Small amounts of sulphur were found in the samples, which is due to the treatment of the titania support with $\mathrm{H}_{2} \mathrm{SO}_{4}$ prior to the deposition of clusters. 
Our XPS analysis of the gold and phosphorous peaks requires these signals to be normalised. The intensity of the gold spectra, i.e. the integral over the peaks fitted to the spectra, has to be normalised as the silicon substrate was, in all cases, not fully covered with the titania particles, i.e. there is a variable loading with titania particles. Normalisation of the phosphorous signal is not required since only the ratio of the phosphorous to gold intensity is of interest. The normalisation of the gold intensity and the calculation of the phosphorous to gold ratio are described in detail in the ESI. $\dagger$

\section{Untreated samples}

The $\mathrm{Au}$ 4f spectra for the $\mathrm{Au}_{8}, \mathrm{Au}_{9}, \mathrm{Au}_{11}$ and $\mathrm{Au}_{101}$ clusters are shown in Fig. 2 for the untreated (i.e. as deposited) clusters (A), the samples washed after deposition (B), and the calcined samples (C). In these spectra, it can be seen that the gold peaks shift towards higher binding energies with decreasing number of atoms in the metal cluster. All four spectra can be fitted with a single peak for each of the $\mathrm{Au} 4 \mathrm{f}_{7 / 2}$ and $\mathrm{Au} 4 \mathrm{f}_{5 / 2}$ components. The peak position and the FWHM are shown in Fig. 3A (as will be discussed below, these peaks are referred to as the gold low binding peak, Au-LBP). The positions of all peaks are at a higher binding energy than that of metallic gold except for $\mathrm{Au}_{101}$. The FWHM of $\mathrm{Au}_{8}, \mathrm{Au}_{9}$ and $\mathrm{Au}_{11}$ is the same within experimental error but are larger than that of $\mathrm{Au}_{101}$. Shifts in the peak position and an increase in the FWHM of the XPS signal of particles consisting of a small number of metal atoms has been attributed in a number of cases to finite size effects and depends on the element constituting the nano-sized object as well as the substrate on which they are deposited. ${ }^{5,46}$ In the case of gold, peak shifts up to +1 to $+1.5 \mathrm{eV},{ }^{4}+0.7 \mathrm{eV},{ }^{35,36}+1 \mathrm{eV}^{3}$ and $+1.1 \mathrm{eV}^{14,47}$ have been previously reported. In all cases, an increase of the FWHM relative to that of bulk gold signal has also been reported. Thus, we attribute the shift of the peak position to the decrease in size of the gold cluster. The peak positions relative to the bulk gold $\mathrm{Au} 4 \mathrm{f}_{7 / 2}$ found here are $+1.1 \mathrm{eV}$ for $\mathrm{Au}_{8}$ and $\mathrm{Au}_{9},+0.7 \mathrm{eV}$ for $\mathrm{Au}_{11}$ and $-0.1 \mathrm{eV}$ for $\mathrm{Au}_{101}$. The position of $\mathrm{Au} 4 \mathrm{f}_{7 / 2}$ of the untreated samples is very close to the peak position of the reference spectra as shown in Fig. 3D. Thus, it can be concluded that the clusters as deposited are not affected by the titania substrate. The position of the $\mathrm{Au} 4 \mathrm{f}_{7 / 2}$ peaks for both the untreated gold clusters and the reference samples are in the range of the $\mathrm{Au} 4 \mathrm{f}_{7 / 2}$ position reported for nanosized gold. As an example, Battistoni et al. ${ }^{44}$ reported that for $\mathrm{Au}_{9}\left(\mathrm{PPh}_{3}\right)_{8}\left(\mathrm{NO}_{3}\right)_{3}$ deposited on steel plates, the $\mathrm{Au} 4 \mathrm{f}_{7 / 2}$ peak lies at $85.2 \mathrm{eV}$ (using the same energy calibration as here). The position of the gold peak as a function of the diameter of the gold cluster is shown in Fig. 3E. The diameter $d$ of the gold cluster is approximated with $d \sim N^{1 / 3}$ where $N$ is the number of gold atoms in the cluster. For such small values of $N$, this approximation is only a rough estimation to the cluster size but even so, Fig. $3 \mathrm{E}$ has similarities to the $1 / d$ relation found for gold nanoparticles by Boyen et al. ${ }^{47}$ Due to its large size $\mathrm{Au}_{101}$ has a binding energy the same as that
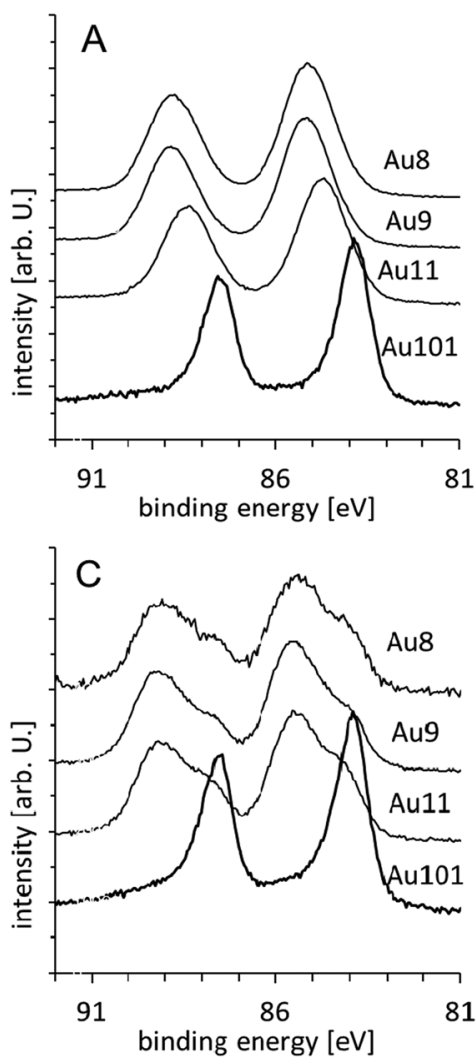

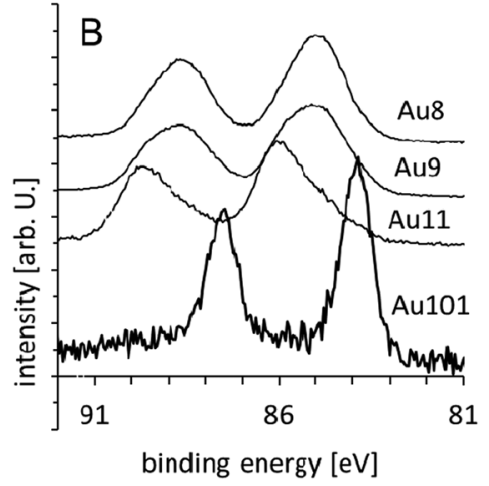

binding energy $[\mathrm{eV}]$ 

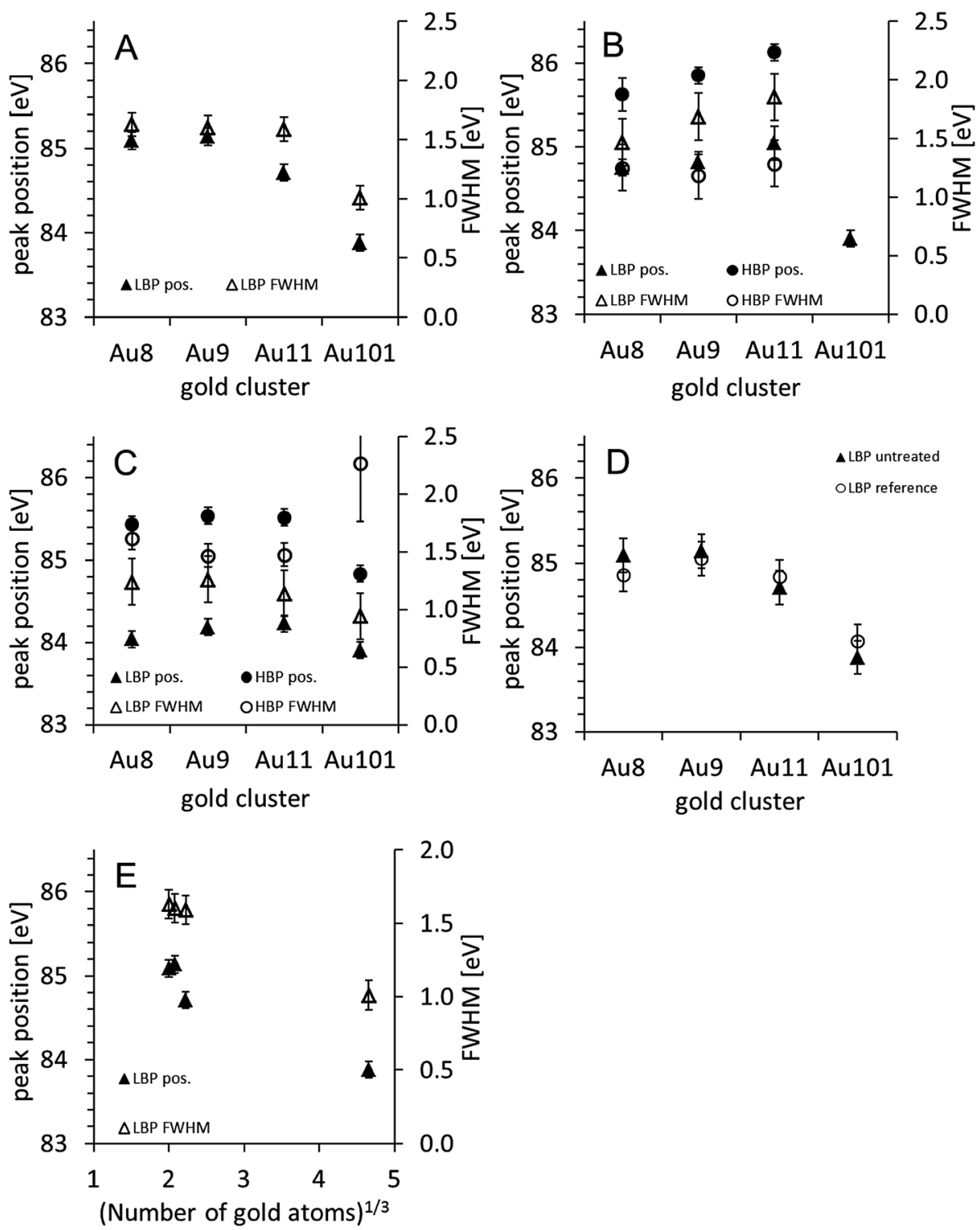

Fig. 3 Position of the Au-LBP and Au-HBP for the (A) untreated, (B) washed and (C) calcined clusters. Note that the Au-LBP peak of the washed Au 101 Cluster in (B) Can be fitted with a single peak. In (D), the peak position of the gold clusters in the reference spectra are shown. In (E) the Au-LBP position and FWHM of the untreated clusters are shown as a function of the diameter, where the number of gold atoms to the power $1 / 3$ is used to approximate the nanoparticle diameter.

of bulk gold (within experimental error). To the best of our knowledge, there is only one previous Au XPS study of a chemically-prepared, atomically precise gold cluster deposited on titania. Chusuei et al. observed a binding energy of $83.9 \mathrm{eV}$ for $\mathrm{Au}_{6}\left(\mathrm{PPh}_{3}\right)_{6}\left(\mathrm{BF}_{4}\right)_{2}$ deposited onto a $\mathrm{TiO}_{2}(110)$ surface, ${ }^{43}$ which is significantly lower than the values we observed. Chusuei et al. used a similar binding energy calibration to that used here.

In Fig. 5A the phosphorous spectra of the untreated $\mathrm{Au}_{8}, \mathrm{Au}_{9}$ and $\mathrm{Au}_{11}$ cluster samples are shown. The phosphorous spectra from the $\mathrm{Au}_{101}$ samples were too weak to reliably separate the spectrum from the background and hence are not shown. The spectra are fitted with two peaks, one at $131.8 \mathrm{eV}$, which we will refer to as the phosphorous low binding energy peak (P-LBP), and another at $133.1( \pm 0.2) \mathrm{eV}$, which we will refer to as the phosphorous high binding energy peak (P-HBP). The position of the P-LBP is the same as that found in the reference samples and also the same as reported by Battistoni et al. ${ }^{44}$ We attribute the P-HBP to oxidised phosphorous which will be justified below. Fig. $6 \mathrm{~A}$ and $5 \mathrm{~B}$ show the ratios of the P-LBP and $\mathrm{P}-\mathrm{HBP}$, respectively, relative to the sum of the $\mathrm{Au} 4 \mathrm{f}_{7 / 2}$ signal. It can be seen that the P-LBP in the untreated samples is the dominant peak (i.e. intensity in Fig. 5A > Fig. 5B for all untreated clusters) and also that the sum of both ratios (Fig. 6C) is close to unity. In the spectrum of $\mathrm{AuPPh}_{3} \mathrm{Cl}$ (not shown here), only the P-LBP is observed with an intensity ratio between phosphorous and gold very close to $1: 1$. Hence, the P-LBP in Fig. 5 is attributed to phosphorous of the ligands bound to gold. Higher binding energies of phosphorous (similar to that of P-HBP) are found for compounds with 
phosphorous bound to oxygen. ${ }^{66}$ Hence, it is concluded that in the untreated support-immobilised clusters there is about one ligand per gold atom and that the nature of the bond between the phosphorous of the ligand and the gold is very similar to that in $\mathrm{AuPPh}_{3} \mathrm{Cl}$.

A molecular representation showing how the $\mathrm{Au}_{9}\left(\mathrm{PPh}_{3}\right)_{8}$ cluster may be deposited onto the titania surface is shown in Fig. 7A, where all ligands remain attached to the cluster core and prevent its contact with the substrate.

\section{Washed samples}

In the $\mathrm{Au} 4 \mathrm{f}$ spectra of the washed samples (Fig. 2B) it is necessary to fit a second, higher energy, gold doublet for $\mathrm{Au}_{8}$, $\mathrm{Au}_{9}$ and $\mathrm{Au}_{11}$ (hereafter referred to as $\mathrm{Au}-\mathrm{HBP}$ ). Fig. 3B shows that the Au-LBP peak positions relative to bulk $\mathrm{Au} 4 \mathrm{f}_{7 / 2}$ are $+0.8 \mathrm{eV}$ for $\mathrm{Au}_{8}$ and $\mathrm{Au}_{9},+1.0 \mathrm{eV}$ for $\mathrm{Au}_{11}$ and $-0.1 \mathrm{eV}$ for $\mathrm{Au}_{101}$ and only marginally different to those of the untreated samples. The FWHMs are the same within the experimental error as those for the Au-LBP in the untreated samples. The Au-HBP is found at $+1.6 \mathrm{eV}$ for $\mathrm{Au}_{8}$, at $+1.9 \mathrm{eV}$ for $\mathrm{Au}_{9},+2.1 \mathrm{eV}$ for $\mathrm{Au}_{11}$. No $\mathrm{Au}-\mathrm{HBP}$ peak is found for $\mathrm{Au}_{101}$. This binding energy is very similar to that of $\mathrm{Au}_{2} \mathrm{O}_{3}$ thin films which shows a shift in binding energy of 1.8 to $2.1 \mathrm{eV} .^{6,31,32}$ Ono et al. have reported shifts in binding energies from about $85 \mathrm{eV}$ to about $87 \mathrm{eV}$ when exposing $1.5 \mathrm{~nm}$ sized gold clusters supported on $\mathrm{TiO}_{2}$ and on $\mathrm{SiO}_{2}$ to oxygen plasma (i.e. about $3 \mathrm{eV}$ higher binding energy than bulk gold). ${ }^{48}$ It is worth noting that $\mathrm{Au}_{101}$ also has a size of about $1.5 \mathrm{~nm}$. Lim et al. reported that gold peaks from gas-phase deposited $\mathrm{Au}_{n}(n=3-20)$ clusters deposited on $\mathrm{SiO}_{2}$ after exposure to atomic oxygen are found at about $86 \mathrm{eV}$ with a shift of about $2 \mathrm{eV}$ relative to the unexposed sample, which they attributed to the formation of gold oxide. ${ }^{6}$

Fig. 4A shows the intensity of both the Au-LBP and Au-HBP. The intensities have been normalised as outlined above and then divided by the intensity of the untreated samples. In general, the total intensity of the peaks is lower than that of the untreated samples (i.e. ratio < 1). Furthermore, it can be seen that in the case of $\mathrm{Au}_{8}$ and $\mathrm{Au}_{9}$ the intensity of the $\mathrm{Au}-\mathrm{LBP}$ is larger than that of the $\mathrm{Au}-\mathrm{HBP}$, while this is reversed for $\mathrm{Au}_{11}$.

As both the position and the FWHM of the Au-LBP are similar to that of the untreated clusters, it can be concluded that this fraction of the gold clusters is not significantly altered in its size and chemical state. We attribute the appearance of the Au-HBP to the formation of $\mathrm{Au}-\mathrm{O}$ bonds involving the gold atoms of the cluster cores as the chemical shifts are significantly larger than that of the untreated clusters. We do not attribute it to fully oxidised gold clusters as the shift in peak position relative to the untreated samples is +0.8 to $+1.1 \mathrm{eV}$ and, thus, significantly less than the $+2 \mathrm{eV}$ shifts reported by others. $^{38,48}$ Whether the shift is due to the formation of partially oxidised gold clusters or due to a strong interaction of the flat (2D), "raft-like" gold cluster core with the $\mathrm{TiO}_{2}$ support cannot be distinguished. Earlier reports suggest that XPS spectra of the ultra-small "raft-like" metal clusters can be strongly affected due to metal-support interactions. ${ }^{67}$ However, it has to be noted that previously oxidation of gold clusters has only been achieved by using a strong oxidising agent. ${ }^{6,31,32}$ In contrast, we have heated the samples to $100{ }^{\circ} \mathrm{C}$ in toluene which is not a strong oxidising environment for the clusters. Hence, the oxidation of the gold with oxygen due to the traces of air dissolved in the liquid phase is less likely than the formation of $\mathrm{Au}-\mathrm{O}$ bonds with oxygen of the titania surface. For the latter, some of the ligands of the gold cluster have to be removed to allow for the formation of $\mathrm{Au}-\mathrm{O}$ bonds. The removal of some or all ligands is considered necessary in the activation of the clusters for catalysis but ${ }^{68}$ to the best of our knowledge this report provides the first ever evidence of the formation of $\mathrm{Au}-\mathrm{O}$ bonds between metal cores of chemically-synthesised atomicallyprecise clusters and oxide support material due to the removal of ligands induced by mild treatment. The removal of ligands also assists in shifts to higher binding energy as ligands are electron donors. In principle, the oxygen signal could be used to further prove this assertion. Unfortunately, the oxygen peaks are dominated by contributions from $\mathrm{SiO}_{2}$ and $\mathrm{TiO}_{2}$ and also, the binding energy of oxygen in $\mathrm{TiO}_{2}$ and gold oxide is very similar and thus would not provide a distinction between these two species.

Alternatively, it could be argued that the Au-HBP is due to the formation of bulk gold oxide after agglomeration of the gold clusters. We consider this option as unlikely for the same reason as before - that the applied treatment is not a strong enough oxidiser to form an oxide layer on bulk gold. Therefore, the overall reduction in gold intensity has to be interpreted as removal of clusters from the surface through the washing procedure.
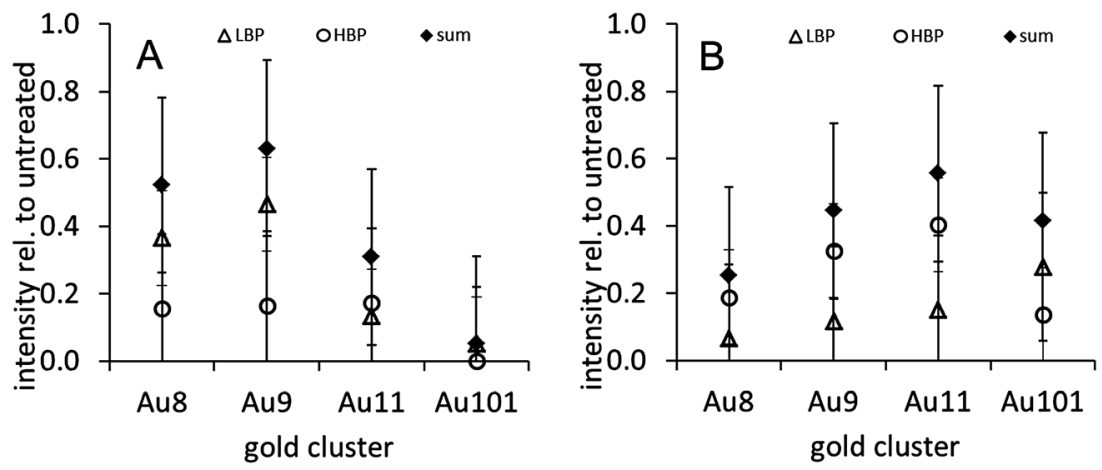

Fig. 4 Ratio of the intensity of the Au-LBP and Au-HBP of (A) washed and (B) calcined clusters, relative to the intensity of the Au peak for the untreated cluster. 

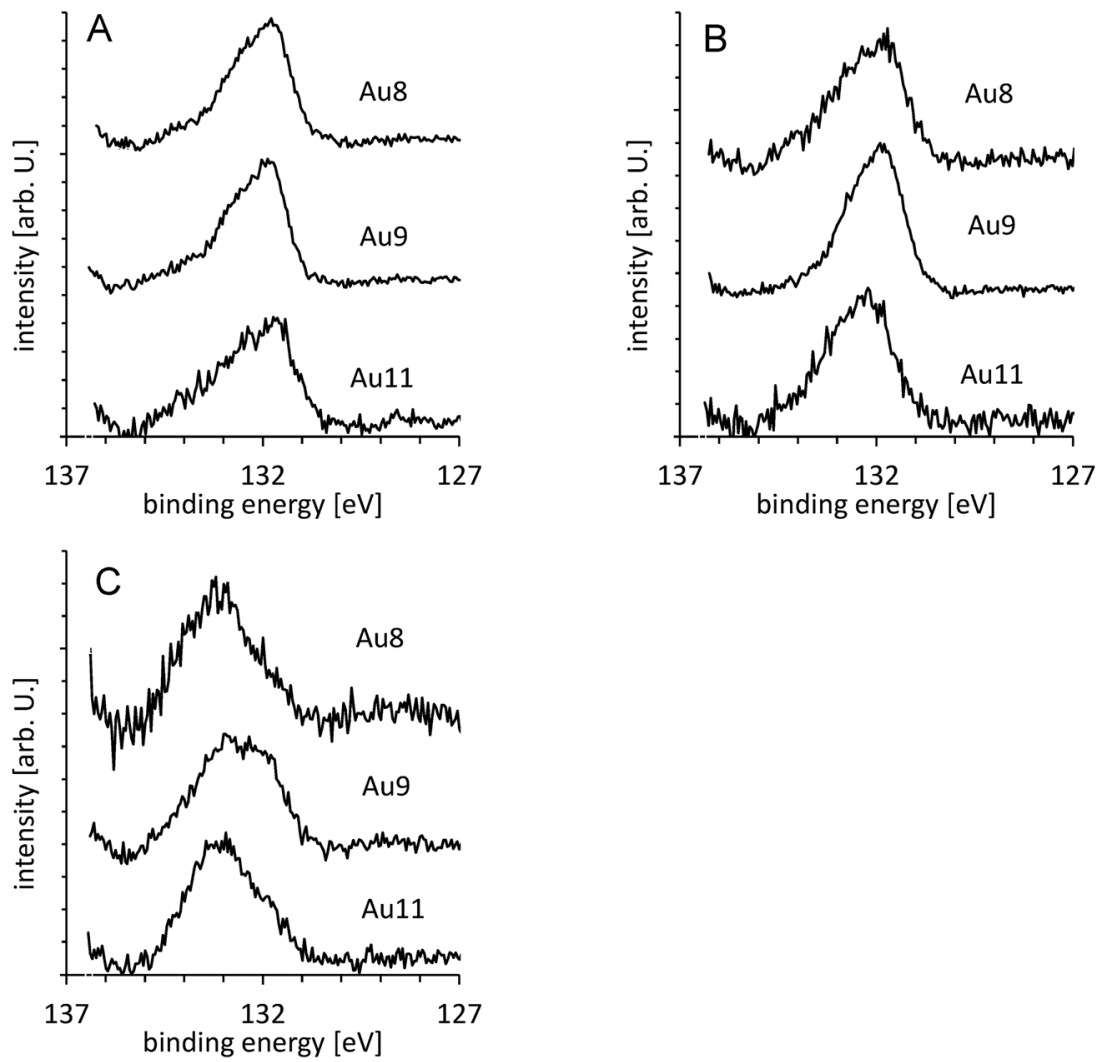

Fig. 5 P XPS spectra of (A) untreated, (B) washed and (C) calcined clusters. The spectrum for $\mathrm{Au}_{101}$ is not shown as the $\mathrm{P}$ signal was too weak to be used for reliable subtraction of the background.

The $\mathrm{Au}_{101}$ clusters show a peak at the same position and with the same FWHM as the untreated sample but with a very significant reduction in intensity. Therefore, it seems that the washing process removes $\mathrm{Au}_{101}$ clusters from the sample but does not change the chemical state of the $\mathrm{Au}_{101}$ cluster core. It has to be noted that $\mathrm{Au}_{101}$ clusters are easiest to remove by washing.

In Fig. $5 \mathrm{~B}$, the $\mathrm{P}$ spectra of the washed $\mathrm{Au}_{8}, \mathrm{Au}_{9}$ and $\mathrm{Au}_{11}$ cluster samples are shown. The spectra show peaks with the same position and FWHM as the untreated samples. Fig. 6A and B show the ratio of the P-LBP and P-HBP, respectively, relative to the sum of the $\mathrm{Au} 4 \mathrm{f}_{7 / 2}$ signal. It can be seen that in the washed samples the P-LBP is the dominant form, which is similar to the spectra of the untreated samples, as seen in Fig. 6C. The sum of both ratios is close to unity for the $\mathrm{Au}_{8}$ and $\mathrm{Au}_{9}$ clusters but is around 1.3 for the $\mathrm{Au}_{11}$ cluster. In the latter case, however, we ascribe this to the relatively large uncertainty in peak area.

It could be argued that for the formation of $\mathrm{Au}-\mathrm{O}$ bonds some ligands have to be removed and hence the $\mathrm{P} / \mathrm{Au}$ intensity should decrease but this is not observed. However, the uncertainty of the phosphorous signal is too large to conclude that this is a contradiction.

A molecular representation showing how the $\mathrm{Au}_{9}\left(\mathrm{PPh}_{3}\right)_{8}$ cluster may interact with the titania surface following washing is shown in Fig. 7B. One of the ligands is removed enabling the cluster core to make contact with the substrate and form $\mathrm{Au}-\mathrm{O}$ bonds, which are observed as Au-HPB. It should be emphasised that Fig. 7B is for illustration only and that we do not have evidence for the exact structure of the cluster core when it is in contact with the substrate.

\section{Calcined samples}

The Au $4 \mathrm{f}$ spectra of the calcined samples are shown in Fig. 2C. Again, it is clear that two doublets are required to fit the experimental spectra. Fig. 3C shows that the positions of the Au-LBP found here relative to bulk $\mathrm{Au} 4 \mathrm{f}_{7 / 2}$ are $0.0 \mathrm{eV}$ for $\mathrm{Au}_{8},+0.2 \mathrm{eV}$ for $\mathrm{Au}_{9}$ and $\mathrm{Au}_{11}$ and $-0.1 \mathrm{eV}$ for $\mathrm{Au}_{101}$ and thus are only marginally different to that of bulk gold. The FWHM for these peaks is $c a .1 .2 \mathrm{eV}$, which is smaller than those of the untreated and the washed samples. Based on the trend of the untreated samples (Fig. 3A and C) we attribute the decrease in binding energy of the Au-LBP after calcination to agglomeration of the clusters. The change in the gold binding energy allows us to conclude that the size of the agglomerated clusters is only marginally smaller than that of untreated $\mathrm{Au}_{101}$, which is also reflected in the FWHM of the peaks as they are only little larger than that of $\mathrm{Au}_{101}$. The Au-HBP relative to bulk $\mathrm{Au} 4 \mathrm{f}_{7 / 2}$ is found at $+1.4 \mathrm{eV}$ for $\mathrm{Au}_{8},+1.5 \mathrm{eV}$ for $\mathrm{Au}_{9}$ and $\mathrm{Au}_{11}$ and $+0.8 \mathrm{eV}$ for $\mathrm{Au}_{101}$. The FWHM of these peaks is 1.5 to $1.6 \mathrm{eV}$ and is similar to that of ultra-small, untreated, supportimmobilised clusters. In the same way as for the washed samples, we attribute the $\mathrm{Au}-\mathrm{HBP}$ to the formation of $\mathrm{Au}-\mathrm{O}$ bonds involving the gold atoms of the cluster cores as the chemical shift is similar to, but slightly $(0.2$ to $0.6 \mathrm{eV})$ smaller than that of the washed samples. In this case, the peak positions are too low in binding 

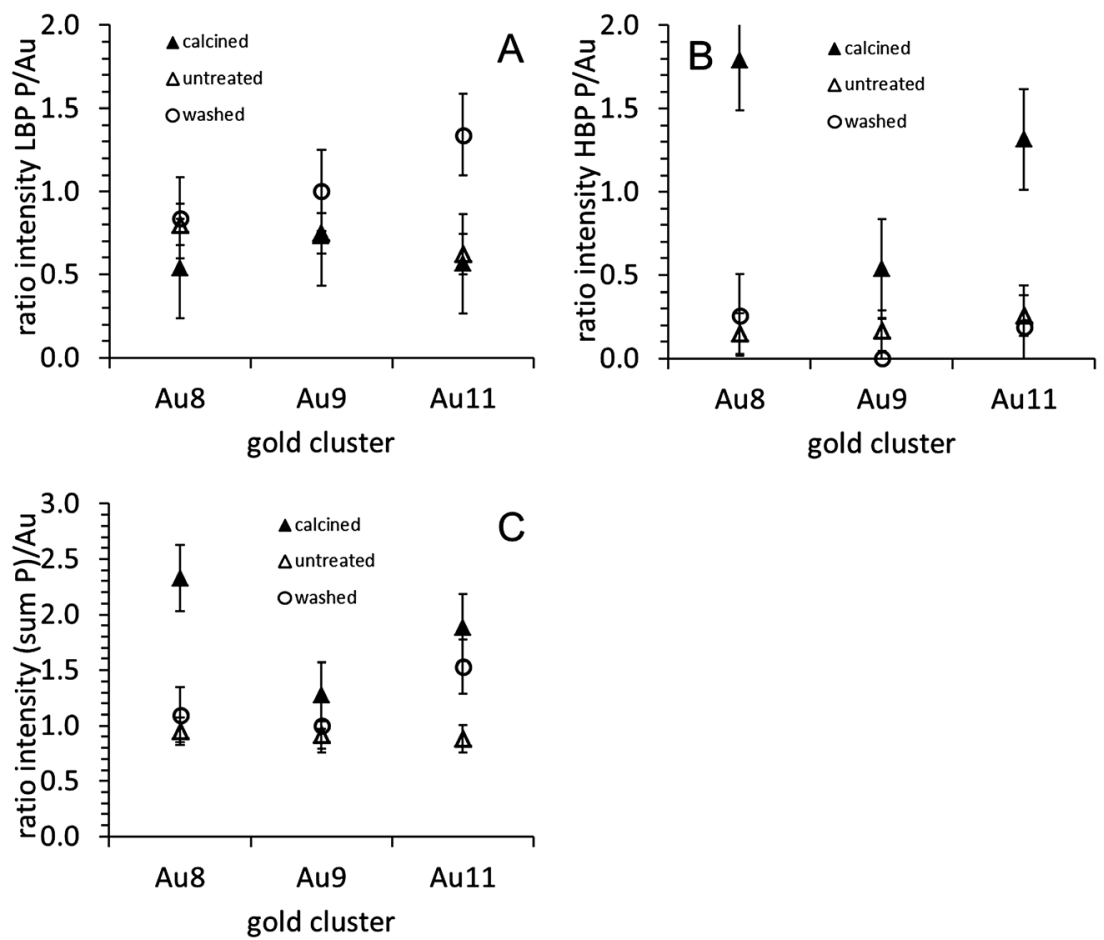

Fig. 6 Ratio of the intensity of (A) the P-LBP, (B) the P-HBP, and (C) the P-HBP + P-LBP, to the sum of the intensity of the respective Au peaks (i.e. both Au-LBP and $\mathrm{Au}-\mathrm{HBP})$.

energy for assigning these peaks to either fully oxidised gold clusters or oxidised bulk gold. Here again, we cannot pinpoint whether the shift is due to the formation of partially oxidised gold clusters or due to a strong interaction of the flat (2D), "raft-like" gold cluster cores with the $\mathrm{TiO}_{2}$ support. Heating at $200{ }^{\circ} \mathrm{C}$ in low vacuum is a somewhat harsher environment than the washing procedure but still cannot be considered as a strong oxidising treatment comparable to oxygen plasma. Also, gold oxide has been found to be unstable at the temperatures applied here. Hence, also in this case the oxidation of the gold with gas phase oxygen is unlikely and the formation of $\mathrm{Au}-\mathrm{O}$ bonds with oxygen of the titania surface has to be considered as being much more likely. The shifts are slightly reduced relative to the washed samples. The reason could be some degree of agglomeration of the clusters that have formed $\mathrm{Au}-\mathrm{O}$ bonds under higher temperature conditions.

The total intensity of gold is significantly reduced compared to the untreated sample as can be seen in Fig. $4 \mathrm{~B}$, with the Au-HBP being more intense than the Au-LBP. Different to washing, calcination does not remove clusters from the support and hence the amount of gold in the sample must be the same as for the untreated samples. Thus, the decrease in gold intensity can only be attributed to agglomeration of the gold cores of the clusters. An increase in gold particle size means that the signal of the gold from the side of the cluster closer to the substrate is attenuated due to the limited mean free path of the photoelectrons. As the amount of gold on the sample is constant, the total gold intensity decreases. This interpretation is supported by the fact that the position of the Au-LBP is close to $84 \mathrm{eV}$.

Fig. 5 shows the phosphorous spectra of the calcined $\mathrm{Au}_{8}$, $\mathrm{Au}_{9}$ and $\mathrm{Au}_{11}$ cluster samples. The spectra clearly illustrate a shift in the peak position compared to the spectra of the untreated samples from the P-LBP to the P-HBP position. A similar shift of the phosphorous peak was reported earlier after calcination of $\mathrm{Au}\left(\mathrm{PPh}_{3}\right)\left(\mathrm{NO}_{3}\right)$ on $\mathrm{TiO}_{2} \cdot{ }^{42}$ Based on XPS results and ${ }^{31} \mathrm{P}-\mathrm{NMR}$, Yuan et al., assigned the change of their sample to either the formation of species where phosphine is bound to a larger metal particle, i.e. the formation of gold clusters surrounded by triphenylphosphine ligands, or the oxidation of phosphorous. Based on our results presented here, we can rule out the first possibility as the untreated $\mathrm{Au}_{n}$ clusters show a lower binding energy of the phosphorous. Hence, we ascribe the $\mathrm{P}$-HBP to oxidised phosphorous species which is also supported by the peak position of phosphorous in phosphorous-doped titania. ${ }^{69}$ Noteworthy, the ratio of the P-LBP and the P-HBP to the sum of the gold $4 \mathrm{f}$ signal (see Fig. $6 \mathrm{~B}$ ) has almost entirely shifted to the P-HBP peak for the $\mathrm{Au}_{8}$ and $\mathrm{Au}_{11}$ clusters and to some extent for the $\mathrm{Au}_{9}$ cluster. The sum of both ratios is larger than unity for the $\mathrm{Au}_{8}$ and $\mathrm{Au}_{11}$ clusters. This finding can be explained by gold clusters agglomerating into larger particles during calcination, consistent with the Au-LBP signal shifting to lower binding energy and the overall decrease in total gold peak intensity. The extraneous ligand $\mathrm{P}$ moves elsewhere onto the surface and becomes oxidised or bonded to the titania surface resulting in an increase in the P-HBP signal (see Fig. 6B). The formation of larger gold particles should reduce the gold signal due to the limited electron mean free path leading to an increase in the total $\mathrm{P}: \mathrm{Au}$ ratio (see Fig. $6 \mathrm{C}$ ) and also to an increase in the P-HBP signal (see Fig. 6B). The amount of "ligand-like" phosphine species (P-LBP) is reduced by $c a$. $50 \%$ due to the maintained presence of a fraction of the non-agglomerated 

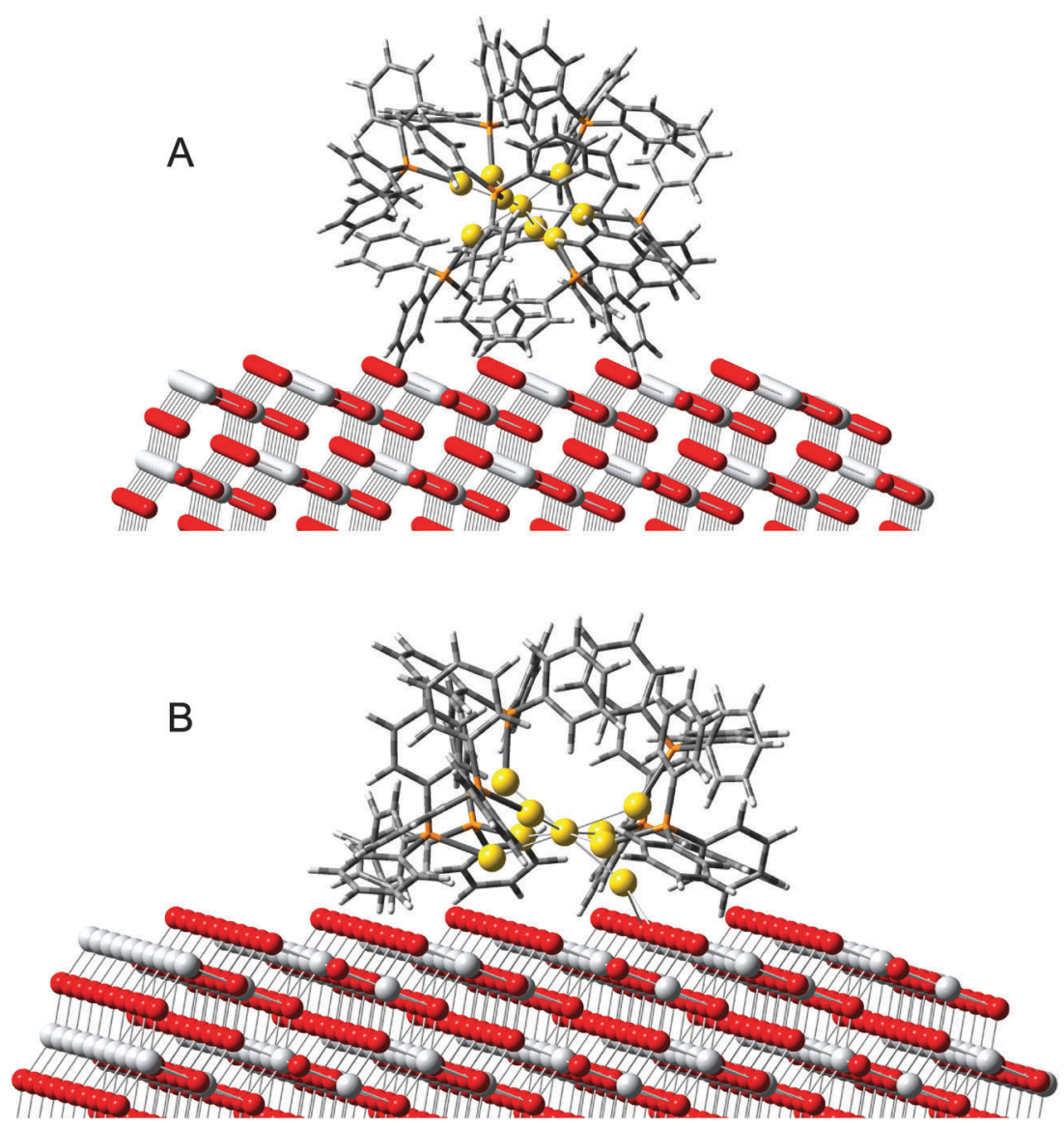

Fig. 7 Schematic illustration of the changes of the clusters due to washing and calcination. In (A) the cluster is shown after deposition $\left(\mathrm{Au}_{9}\left(\mathrm{PPh}_{3}\right)_{8}\right)$. The ligands are attached to the cluster core and the core is not in contact with the substrate. In (B), one of the ligands is removed (i.e. $\left.\mathrm{Au}_{9}\left(\mathrm{PPh}_{3}\right)_{7}\right)$ and the core is in contact with the substrate. (Note: whilst these figures are based upon the crystal structures of the Aug cluster and anatase (101) surface separately, the relative position and orientation of the cluster-surface interaction does not involve any geometric or energy minimisation process. They should therefore be regarded as crude representations of a possible interaction that is consistent with the XPS data. It should also be noted that the counter ion is not shown. Furthermore, it must be emphasised that the exact number of ligands removed is unknown and may be more than shown here.)

clusters even in calcined samples (see Fig. 6A). This interpretation is supported for $\mathrm{Au}_{8}$ and $\mathrm{Au}_{11}$ by the changes in the phosphorous signal shown in Fig. 6. To a lesser degree the interpretation is also supported for $\mathrm{Au}_{9}$. However, the removal of the ligands from the cluster and their oxidation seems to be less efficient for $\mathrm{Au}_{9}$ compared to $\mathrm{Au}_{8}$ and $\mathrm{Au}_{11}$, as the P-LBP is stronger in the case of $\mathrm{Au}_{9}$ compared to $\mathrm{Au}_{8}$ and $\mathrm{Au}_{11}$. In summary, it can be concluded that the phosphorous of the ligands oxidises during calcination and is most likely removed from the clusters but remains in an oxidised form on the surface. The changes of the clusters deposited on the titania particles due to the calcination procedure applied are illustrated in Fig. 7. In terms of the molecular representation in Fig. 7B, although calcining of the sample does lead to removal of ligands, it is also observed that agglomeration of the gold core occurs.

\section{Conclusions}

We have investigated chemically synthesised, atomically precise gold clusters deposited on titania nanoparticles using XPS. Subsequent to their immobilisation on the titania support, the gold clusters have been treated with washing and calcination to remove the phosphine ligands from the gold cluster core. XPS has been used as a tool to determine changes in the chemical nature, composition, and to estimate changes in the size of the gold clusters. Upon treatment three changes of the clusters have been found: firstly, removal of the phosphine ligands from the Au clusters and interaction of the cluster core with the substrate, secondly, partial agglomeration of the Au clusters and thirdly, oxidation of the phosphine ligands. The removal of the ligands is most likely an essential step to make the clusters catalytically active and their interaction with the substrate will affect their catalytic activity since it influences their charge state. Agglomeration of the clusters will also impact the catalytic properties of the deposited clusters. It is known that small clusters show size specific catalytic activity ${ }^{8,9}$ which is lost when they agglomerate. However, only partial agglomeration is found here and a portion of the clusters are not affected. As the ligands are at least partially removed, the non-agglomerated clusters could be involved in catalytic reactions. The interaction with the substrate could also affect the catalytic activity.

We find that upon depositions, untreated clusters remain virtually unchanged and can be described as ultra-small clusters 
with decreasing size from $\mathrm{Au}_{101}$ to $\mathrm{Au}_{8}$, as indicated by positions and widths of the gold and phosphorus peaks in the XPS spectra. The peak positions in the gold XPS spectra shift systematically in agreement with the size of the clusters determined by X-ray diffraction immediately after their synthesis.

Washing of the support-immobilised cluster with toluene at $100{ }^{\circ} \mathrm{C}$ leads to the removal of a fraction of the clusters from the titania surface. Of those clusters left on the titania surface some remain virtually unchanged. The other fraction shows formation of $\mathrm{Au}-\mathrm{O}$ bonds, most likely to the oxygen of the titania surface, correlating with at least partial removal of ligands. No significant agglomeration of the cluster cores is evident from the observed XPS spectra for this fraction.

Calcination of the support-immobilised clusters has two effects. First, the ultra-small clusters aggregate to form slightly larger gold particles that are still protected by phosphine ligands. The average size of the aggregated clusters can be estimated to be marginally smaller than that of untreated $\mathrm{Au}_{101}$. Second, a fraction of gold clusters forms $\mathrm{Au}-\mathrm{O}$ bonds, again most likely to the oxygen of the titania surface, which coincides with the loss of phosphine ligands and formation of oxidised phosphorous species. Of this fraction of clusters, at least a portion does not show agglomeration.

\section{Acknowledgements}

This work was supported by a grant of the Australian Synchrotron (AS112/SXR/3576), the MacDiarmid Institute and the College of Science at the Canterbury University. The XPS measurements were undertaken on the soft X-ray beamline at the Australian Synchrotron, Victoria, Australia.

\section{References}

1 P. L. J. Gunter, J. W. Niemantsverdriet, F. H. Ribeiro and G. A. Somorjai, Catal. Rev.: Sci. Eng., 1997, 39, 77-168.

2 F. H. Ribeiro, G. A. Somorjai, G. Wedler, B. C. Gates, C. T. Campbell, C. Xu, D. W. Goodman, A. Zecchina, D. Scarano, S. Bordiga, A. Cimino, F. S. Stone, Y. Iwasawa, M. A. Barteau, J. M. Vohs and J. P. Vigneron, in Handbook of Heterogeneous Catalysis, Wiley-VCH Verlag GmbH, 2008, pp. 771-908.

3 M. S. Chen and D. W. Goodman, Chem. Soc. Rev., 2008, 37, 1860-1870.

4 M. Büttner and P. Oelhafen, Surf. Sci., 2006, 600, 1170-1177.

5 Y. Kitsudo, A. Iwamoto, H. Matsumoto, K. Mitsuhara, T. Nishimura, M. Takizawa, T. Akita, Y. Maeda and Y. Kido, Surf. Sci., 2009, 603, 2108-2114.

6 D.-C. Lim, C.-C. Hwang, G. Gantefor and Y. D. Kim, Phys. Chem. Chem. Phys., 2010, 12, 15172-15180.

7 U. Heiz and W.-D. Schneider, Crit. Rev. Solid State Mater. Sci., 2001, 26, 251-290.

8 S. Kunz, K. Hartl, M. Nesselberger, F. F. Schweinberger, G. Kwon, M. Hanzlik, K. J. J. Mayrhofer, U. Heiz and M. Arenz, Phys. Chem. Chem. Phys., 2010, 12, 10288-10291.
9 B. Yoon, H. Häkkinen, U. Landman, A. S. Wörz, J.-M. Antonietti, S. Abbet, K. Judai and U. Heiz, Science, 2005, 307, 403-407.

10 R. Schlögl, M. Che, O. Clause, C. Marcilly, C. Louis, H. Knöinger, E. Teglauer, W. Keim, B. Drieben-Hölscher, J. W. Geus, A. J. van Dillen and J. Barbier, in Handbook of Heterogeneous Catalysis, Wiley-VCH Verlag $\mathrm{GmbH}, 2008$, pp. 138-264.

11 R. M. Crooks, M. Q. Zhao, L. Sun, V. Chechik and L. K. Yeung, Acc. Chem. Res., 2001, 34, 181-190.

12 M. Faraday, Philos. Trans. R. Soc. London, 1857, 147, 145-181.

13 A. B. Hungria, R. Raja, R. D. Adams, B. Captain, J. M. Thomas, P. A. Midgley, V. Golovko and B. F. G. Johnson, Angew. Chem., Int. Ed., 2006, 45, 4782-4785.

14 M. Turner, V. B. Golovko, O. P. H. Vaughan, P. Abdulkin, A. Berenguer-Murcia, M. S. Tikhov, B. F. G. Johnson and R. M. Lambert, Nature, 2008, 454, 981-983.

15 R. Raja, V. B. Golovko, J. M. Thomas, A. Berenguer-Murcia, W. Z. Zhou, S. H. Xie and B. F. G. Johnson, Chem. Commun., 2005, 2026-2028.

16 Y. M. Liu, H. Tsunoyama, T. Akita and T. Tsukuda, Chem. Commun., 2010, 46, 550-552.

17 Y. M. Liu, H. Tsunoyama, T. Akita, S. H. Xie and T. Tsukuda, ACS Catal., 2011, 1, 2-6.

18 J. A. Lopez-Sanchez, N. Dimitratos, C. Hammond, G. L. Brett, L. Kesavan, S. White, P. Miedziak, R. Tiruvalam, R. L. Jenkins, A. F. Carley, D. Knight, C. J. Kiely and G. J. Hutchings, Nat. Chem., 2011, 3, 551-556.

19 Y. Zhu, H. Qian and R. Jin, J. Mater. Chem., 2011, 21, 67936799.

20 C. C. Chusuei, X. Lai, K. A. Davis, E. K. Bowers, J. P. Fackler and D. W. Goodman, Langmuir, 2001, 17, 4113-4117.

21 M. Haruta, S. Tsubota, T. Kobayashi, H. Kageyama, M. J. Genet and B. Delmon, J. Catal., 1993, 144, 175-192.

22 R. Meyer, C. Lemire, S. Shaikhutdinov and H. Freund, Gold Bull., 2004, 37, 72-124.

23 M. Valden, X. Lai and D. W. Goodman, Science, 1998, 281, 1647-1650.

24 A. A. Herzing, C. J. Kiely, A. F. Carley, P. Landon and G. J. Hutchings, Science, 2008, 321, 1331-1335.

25 N. Yi, R. Si, H. Saltsburg and M. Flytzani-Stephanopoulos, Appl. Catal., B, 2010, 95, 87-92.

26 N. Yi, R. Si, H. Saltsburg and M. Flytzani-Stephanopoulos, Energy Environ. Sci., 2010, 3, 831-837.

27 W. Huang, H.-J. Zhai and L.-S. Wang, J. Am. Chem.Soc., 2010, 132, 4344-4351.

28 Z. Jiang, W. Zhang, L. Jin, X. Yang, F. Xu, J. Zhu and W. Huang, J. Phys. Chem. C, 2007, 111, 12434-12439.

29 M. Walter, J. Akola, O. Lopez-Acevedo, P. D. Jadzinsky, G. Calero, C. J. Ackerson, R. L. Whetten, H. Grönbeck and H. Häkkinen, Proc. Natl. Acad. Sci. U. S. A., 2008, 105, 9157-9162.

30 S. Porsgaard, P. Jiang, F. Borondics, S. Wendt, Z. Liu, H. Bluhm, F. Besenbacher and M. Salmeron, Angew. Chem., Int. Ed., 2011, 50, 2266-2269.

31 K. Juodkazis, J. Juodkazytè, V. Jasulaitienè, A. Lukinskas and B. Šebeka, Electrochem. Commun., 2000, 2, 503-507. 
32 B. Koslowski, H. G. Boyen, C. Wilderotter, G. Kästle, P. Ziemann, R. Wahrenberg and P. Oelhafen, Surf. Sci., 2001, 475, 1-10.

33 V. Borman, M. Pushkin, V. Tronin and V. Troyan, J. Exp. Theor. Phys., 2010, 110, 1005-1025.

34 S. Zafeiratos and S. Kennou, Surf. Sci., 1999, 443, 238-244.

35 Y. Lykhach, J. Plšek, I. Spirovová and Z. Bastl, Collect. Czech. Chem. Commun., 2003, 68, 1791-1804.

36 S. B. DiCenzo, S. D. Berry and E. H. Hartford, Jr., Phys. Rev. B: Condens. Matter Mater. Phys., 1988, 38, 8465-8468.

37 D. M. Cox, W. Eberhardt, P. Fayet, Z. Fu, B. Kessler, R. D. Sherwood, D. Sondericker and A. Kaldor, Z. Phys. D: At., Mol. Clusters, 1991, 20, 385-386.

38 D. C. Lim, R. Dietsche, G. Ganteför and Y. D. Kim, Chem. Phys. Lett., 2008, 457, 391-395.

39 D. C. Lim, R. Dietsche, G. Ganteför and Y. D. Kim, Chem. Phys., 2009, 359, 161-165.

40 D. C. Lim, R. Dietsche, G. Ganteför and Y. D. Kim, Appl. Surf. Sci., 2009, 256, 1148-1151.

41 W. W. Weare, S. M. Reed, M. G. Warner and J. E. Hutchison, J. Am. Chem. Soc., 2000, 122, 12890-12891.

42 Y. Yuan, K. Asakura, A. P. Kozlova, H. Wan, K. Tsai and Y. Iwasawa, Catal. Today, 1998, 44, 333-342.

43 C. C. Chusuei, X. Lai, K. A. Davis, E. K. Bowers, J. P. Fackler and D. W. Goodman, Langmuir, 2001, 17, 4113-4117.

44 C. Battistoni, G. Mattogno, R. Zanoni and L. Naldini, J. Electron Spectrosc. Relat. Phenom., 1982, 28, 23-31.

45 C. Battistoni, G. Mattogno, F. Cariati, L. Naldini and A. Sgamellotti, Inorg. Chim. Acta, 1977, 24, 207-210.

46 P. M. T. M. Van Attekum, J. W. A. Van der Velden and J. M. Trooster, Inorg. Chem., 1980, 19, 701-704.

47 H. G. Boyen, A. Ethirajan, G. Kästle, F. Weigl, P. Ziemann, G. Schmid, M. G. Garnier, M. Büttner and P. Oelhafen, Phys. Rev. Lett., 2005, 94, 016804.

48 L. K. Ono and B. Roldan Cuenya, J. Phys. Chem. C, 2008, 112, 4676-4686.

49 C. N. R. Rao, V. Vijayakrishnan, H. N. Aiyer, G. U. Kulkarni and G. N. Subbanna, J. Phys. Chem., 1993, 97, 11157-11160.

50 A. K. Santra and D. W. Goodman, J. Phys.: Condens. Matter, 2003, 15, R31.
51 L. Ellis-Gibbings, V. Johansson, R. B. Walsh, L. Kloo, J. S. Quinton and G. G. Andersson, Langmuir, 2012, 28, 9431-9439.

52 M. Murdoch, G. I. N. Waterhouse, M. A. Nadeem, J. B. Metson, M. A. Keane, R. F. Howe, J. Llorca and H. Idriss, Nat. Chem., 2011, 3, 489-492.

53 Y.-Y. Fong, B. R. Visser, J. R. Gascooke, B. C. C. Cowie, L. Thomsen, G. F. Metha, M. A. Buntine and H. H. Harris, Langmuir, 2011, 27, 8099-8104.

54 B. C. C. Cowie, A. Tadich and L. Thomsen, AIP Conf. Proc., 2010, 1234, 307-310.

55 D. A. Shirley, Phys. Rev. B: Solid State, 1972, 5, 4709-4714.

56 J. Végh, J. Electron Spectrosc. Relat. Phenom., 2006, 151, 159-164.

57 H. P. Hughes and J. A. Scarfe, J. Phys.: Condens. Matter, 1996, 8, 1421.

58 J. F. Moulder, W. F. Stickle, P. E. Sobol and K. D. Bomben, Handbook of X-ray Photoelectron Spectroscopy, Physical Electronics, Inc., Eden Prairie, 1995.

59 J. J. Yeh and I. Lindau, At. Data Nucl. Data Tables, 1985, 32, 1-155.

60 F. Wen, U. Englert, B. Gutrath and U. Simon, Eur. J. Inorg. Chem., 2008, 106-111.

61 D. Briggs and M. P. Seah, Practical surface analysis, Wiley, Chichester, New York, Aarau, 1990.

62 K. Asami, J. Electron Spectrosc. Relat. Phenom., 1976, 9, 469-478.

63 K. Dückers and H. P. Bonzel, Surf. Sci., 1989, 213, 25-48.

64 T. Darrah Thomas and P. Weightman, Phys. Rev. B: Condens. Matter Mater. Phys., 1986, 33, 5406-5413.

65 M. P. Seah, G. C. Smith and M. T. Anthony, Surf. Interface Anal., 1990, 15, 293-308.

66 C. A. Strydom and H. J. Strydom, Inorg. Chim. Acta, 1989, 159, 191-195.

67 A. Y. Stakheev and L. M. Kustov, Appl. Catal., A, 1999, 188, 3-35.

68 A. Kulkarni, R. J. Lobo-Lapidus and B. C. Gates, Chem. Commun., 2010, 46, 5997-6015.

69 L. Lin, R. Y. Zheng, J. L. Xie, Y. X. Zhu and Y. C. Xie, Appl. Catal., B, 2007, 76, 196-202. 